\title{
Everolimus enhances cellular cytotoxicity of lapatinib via the eukaryotic elongation factor-2 kinase pathway in nasopharyngeal carcinoma cells
}

This article was published in the following Dove Press journal:

OncoTargets and Therapy

II October 2016

Number of times this article has been viewed

\author{
Lin Liu ${ }^{1, *}$ \\ Zhi-Hui Wangl,* \\ Jun Han' \\ Con Tang ${ }^{2}$ \\ Nan Chen' \\ Zhong Lin' \\ Pei-Jian Pengl,* \\ 'Department of Medical Oncology, \\ ${ }^{2}$ Department of Surgical Oncology, \\ The Fifth Affiliated Hospital of \\ Sun-Yat-Sen University, Zhu Hai, \\ Guangdong Province, People's \\ Republic of China \\ *These authors contributed equally \\ to this work
}

Background: Nasopharyngeal carcinoma (NPC) has a high relapse and metastatic rates; hence, development of new therapeutics is an immediate requirement. Lapatinib and everolimus have been demonstrated to be effective in the treatment of several carcinomas. This preclinical study aimed to investigate the effect and mechanism of lapatinib combined with everolimus on NPC cells.

Methods: The Cell Counting Kit 8 and colony formation assay were used to detect the effect of lapatinib alone or lapatinib combined with everolimus on the growth and proliferation of cells. Apoptosis was tested by flow cytometry and was further confirmed by western blot. The targets of lapatinib and the effects of lapatinib or everolimus on the eukaryotic elongation factor-2 (eEF-2) kinase pathway were analyzed by western blot, which also evaluated autophagy activity.

Results: Lapatinib inhibited the cellular viability and colony formation in NPC cells. At 24-72 h, the average half maximal inhibitory concentration $\left(\mathrm{IC}_{50}\right)$ values of lapatinib were ranging from 3 to $5 \mu \mathrm{M}$. This study further found that lapatinib induced both apoptosis and autophagy in NPC cells, and this autophagic activity was described as type II programmed cell death via an eEF-2 kinase-dependent pathway. In addition, augmentation of lapatinib-induced autophagy by mammalian target of rapamycin (mTOR) inhibitor everolimus enhanced the cytocidal effect of lapatinib in NPC cells via the mTOR/S6 kinase/eEF-2 kinase pathway.

Conclusion: This study reveals that everolimus can sensitize NPC cells to lapatinib by the activation of eEF-2 kinase and provides a potential model of combination therapy.

Keywords: lapatinib, eEF-2K, everolimus, nasopharyngeal carcinoma

\section{Introduction}

Nasopharyngeal carcinoma (NPC), arising from the epithelium lining of the posterior nasopharynx, is a highly invasive and metastatic head and neck cancer. It is common especially in southern China and Southeast Asia with an incidence rate of 15-30/100,000. . $^{2}$ The median survival is only 5-11 months for patients with metastatic or advanced NPC. ${ }^{3}$ The relapse rate of metastatic NPC is as high as $82 \%,{ }^{4}$ even with aggressive radiation and chemotherapy treatment. The poor prognosis is due to high recurrence and metastatic rates. Consequently, new therapeutics against NPC are urgently needed.

Activation of the epidermal growth factor receptor (EGFR) pathway promotes the invasive of NPC. ${ }^{5}$ Findings suggest that the EGFRs (four related members) may be good molecular targets for various malignancies, including NPC. ${ }^{6}$

Lapatinib is a reversible tyrosine kinase inhibitor that targets both EGFR and HER-2. ${ }^{7}$ Overexpression of EGFR/HER-2 indicates poor prognosis in breast cancer, and lapatinib was approved as a therapy for this cancer. In addition, many preclinical
Correspondence: Pei-Jian Peng Department of Medical Oncology, The Fifth Affiliated Hospital of Sun-YatSen University, 52 Mei Hua Road East, Zhu Hai 519000, Guangdong Province, People's Republic of China

Tel +867562528020

Fax +867562528045

Email pengpjian@।63.com 
and clinical studies on the effectiveness of lapatinib against various solid tumors, including breast, lung, hepatocellular, head and neck, vulva, colon, prostate, ovarian, and gastric cancers are currently ongoing. ${ }^{7-10}$ However, the role of lapatinib in the treatment of NPC remains unclear.

Many antitumor agents induce apoptosis in cancer; aside from this, autophagy, which is characterized by support cell survival, in some instances will cause cell death. ${ }^{11}$ Recently, autophagy was found to be activated by lapatinib, yet the exact function of autophagy on the cytocidal activity of lapatinib in NPC remains unknown. ${ }^{12,13}$

Eukaryotic elongation factor-2 (eEF-2) kinase, phosphorylating the only known substrate eEF-2 on Thr56 to regulate protein synthesis, is a unique enzyme and is upregulated in various malignant neoplasms. ${ }^{14}$ Recently, some investigations have reported that eEF-2 kinase can regulate autophagy under environmental or metabolic stress. ${ }^{15,16}$

Since mammalian target of rapamycin (mTOR) and S6 kinase are involved in a pathway that controls the activity of eEF-2 kinase, this study aimed to determine whether eEF-2 kinase participates in lapatinib-induced autophagy and how to exploit the mechanisms to increase cytocidal efficacy.

\section{Materials and methods}

\section{Cell lines and culture}

Two poorly differentiated human NPC cell lines, CNE-2 and HONE-1, were generously provided by State Key Laboratory of Oncology in South China. Both the cell lines were cultivated in RPMI-1640 medium (Thermo Fisher Scientific, Waltham, MA, USA) supplemented with 10\% fetal bovine serum (Thermo Fisher Scientific) and antibiotics $(10,000 \mathrm{U} / \mathrm{mL}$ penicillin and $10 \mu \mathrm{g} / \mathrm{mL}$ streptomycin). Cells were maintained in a humidified atmosphere at $37^{\circ} \mathrm{C}$ with $5 \%$ carbon dioxide.

\section{Inhibitors}

All inhibitors, including lapatinib, hydroxychloroquine (HCQ) sulfate, and everolimus, were obtained from Selleck Chemicals (USA), stored at $-20^{\circ} \mathrm{C}$ and diluted in fresh culture medium before use. The working solutions of lapatinib and everolimus were dissolved in dimethyl sulfoxide (DMSO). The concentration of DMSO in the final solution did not exceed $1 \%(\mathrm{v} / \mathrm{v})$. The stock solution of HCQ sulfate was formulated to $50 \mathrm{mM}$ with ultrapure water.

\section{Cell viability analysis}

CNE-2 and HONE-1 cells were seeded in 96-well plates at a density of $2.0-4.0 \times 10^{3}$ cells/well and incubated with different inhibitors at various dilutions for 24,48 , and $72 \mathrm{~h}$.
Cell viability was tested by Cell Counting Kit-8 (CCK-8 Kit; Dojindo Co., Japan) according to the manufacturer's instructions. The viability of the control group (DMSO vehicle treated) was normalized to $100 \%$. Cell survival rate $(\%)=(\mathrm{OD}$ value of treatment group/OD value of control group) $\times 100 \%$. The $\mathrm{IC}_{50}$ values, defined as the concentration resulting in 50\% cell growth inhibition after treating with lapatinib or everolimus compared with control cells, were calculated using GraphPad Prim 5 software (GraphPad Software, San Diego, CA, USA). All the experiments were performed in triplicate.

\section{Colony formation assay}

Cells, suspended at a density of 200-400 cells per well, were seeded into 6-well plates and incubated with either inhibitor alone or in combination at the indicated concentrations. The medium was replaced with inhibitors every 3 days. After 7-14 days, cells were stained with 1\% methylene blue trihydrate for $20 \mathrm{~min}$.

\section{Western blot analysis}

Cells were lysed in RIPA lysis buffer (Thermo, Hercules, CA, USA), which contained protease (Sigma-Aldrich Co., St Louis, MO, USA) and phosphatase inhibitor cocktails PhosSTOP (Roche, Mannheim, Germany), for $30 \mathrm{~min}$ on ice. The lysates were centrifuged at $13,000 \mathrm{rpm}$ for $20 \mathrm{~min}$ at $4^{\circ} \mathrm{C}$. The concentration was determined by the Thermo Protein Assay Reagent (Thermo). A total of 30-100 $\mu \mathrm{g}$ protein was loaded into each well of a $6 \%-15 \%$ sodium dodecyl sulfate-polyacrylamide gel. The resolved proteins were electrophoretically transferred to polyvinylidene difluoride membranes (Bio-Rad Laboratories, Hercules, CA, USA) and immunoblotted sequentially with primary and secondary antibodies. The membranes were incubated overnight at $4{ }^{\circ} \mathrm{C}$ with the following primary antibodies: PhosphomTOR (Ser2481), EGF Receptor (D38BI), phospho-EGFR (Tyr1068), HER2/Erb2 (D8F12), Phospho-HER2/ErbB2 (Tyr1221/1222) (6B12), eEF2K, phospho-eEF2 (Thr56), eEF2, Cleaved PARP (Asp214) (D64E10), Phospho-S6 Ribosomal Protein (Ser235/236), GAPDH, LC3 A/B. All antibodies were obtained from Cell Signaling Technology (Danvers, MA, USA). After three washes, the membrane was further incubated with horseradish peroxidase-conjugated goat anti-rabbit secondary antibodies (1:2000, Santa Cruz Biotechnology Inc., Dallas, TX, USA) at room temperature for $1 \mathrm{~h}$. Bands were detected using super signal West Dura Luminol/Enhancer solution (Thermo) following the manufacturer's instructions. Finally, ImageJ software was used to measure the gray value of protein, and the gray value 
of GAPDH was normalized to $100 \%$. Then, the density of band $=$ the gray value of sample/the gray value of GAPDH (internal reference).

\section{Flow cytometry}

Cells were seeded at a density of $1.0 \times 10^{5}$ cells/well into a sixwell plate and then treated with lapatinib for $48 \mathrm{~h}$. Annexin V-APC/7-AAD Apoptosis Detection kit (KGA1023-1026; KeyGEN, Nanjing, People's Republic of China) was used to stain the apoptosis cells. Apoptosis was measured by flow cytometry (FC500; Beckman Coulter, Brea, CA, USA). Annexin V-APC-positive cells (both 7-AAD-negative and 7-AAD-positive) were defined as apoptotic cells. Three independent experiments were performed, and the results were displayed as histograms.

\section{RNA-mediated gene knockdown}

Lentivirus-based short hairpin RNA (shRNA) targeting eEF-2 kinase was obtained from Genechem Co., Ltd. (Shanghai, People's Republic of China). Nonsilencing, scrambled (nontargeting), and green fluorescent protein-specific constructs were used as nontargeting shRNA controls. Transfection was performed according to the manufacturer's protocol.

\section{Statistical analysis}

GraphPad Prim 5 software (GraphPad Software Inc., La Jolla, CA, USA) was used for all data analyses. All the experiments were performed in triplicate. The independent results were expressed as mean value \pm standard deviation of the mean. The differences between the inhibitors alone, in combination, and controls were analyzed by a two-tailed Student's $t$-test and combination. $P<0.05$ was considered statistically significant.

\section{Results}

\section{Inhibitory effects of lapatinib on viability and colony formation in human NPC cells} Two kinds of poorly differentiated NPC cell lines, CNE-2 and HONE-1 cells, coexpressing EGFR and HER-2, were tested for lapatinib-induced cytotoxicity. Western blot analysis showed that lapatinib inhibited EGFR and HER-2 phosphorylation in CNE-2 and HONE-1 cells (Figure 1A). Cell viability was then
A

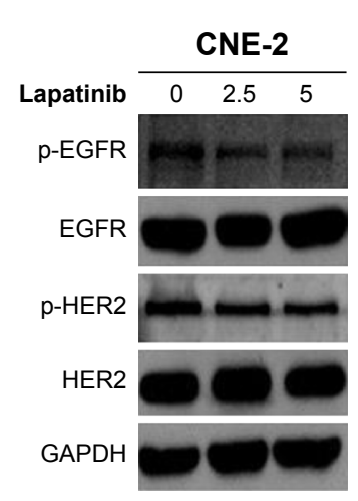

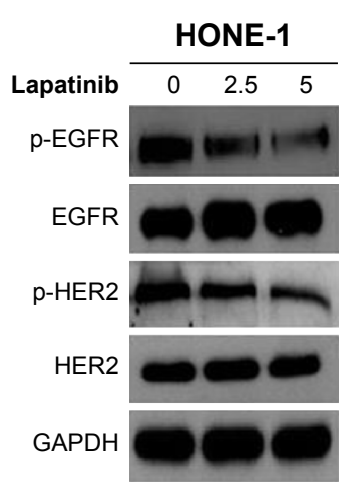

B

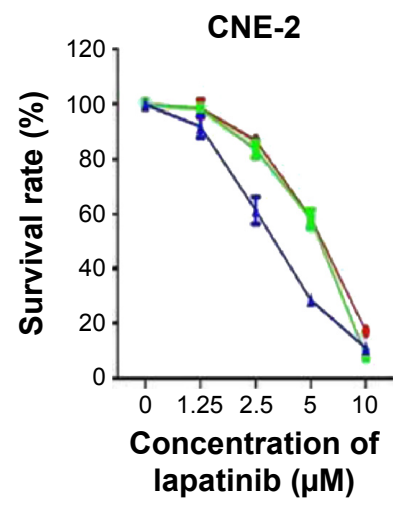

\section{HONE-1}

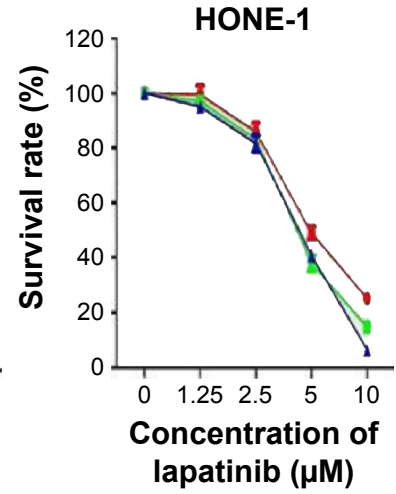

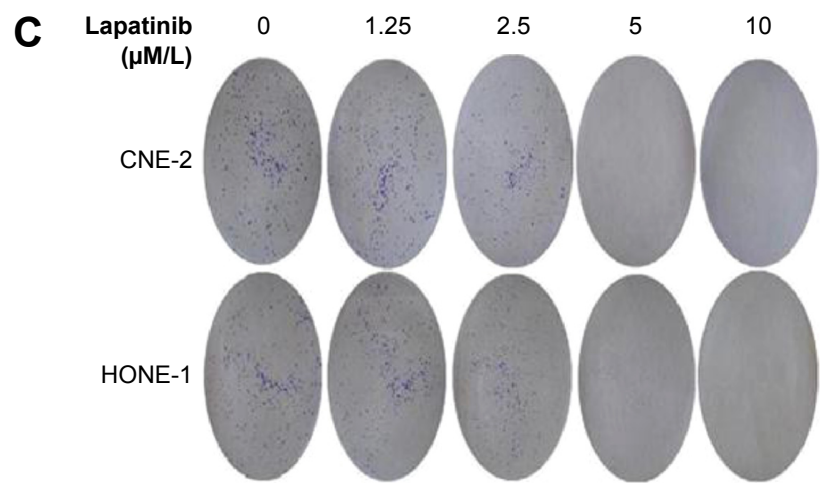

D

\begin{tabular}{lll}
\hline Time (h) & \multicolumn{2}{l}{$\mathrm{IC}_{50}(\boldsymbol{\mu M})$} \\
\cline { 2 - 3 } & $\mathrm{CNE}-2$ & HONE-1 \\
\hline 24 & $5.56 \pm 0.09$ & $5.38 \pm 0.18$ \\
48 & $5.45 \pm 0.17$ & $4.36 \pm 0.09$ \\
72 & $3.24 \pm 0.15$ & $4.30 \pm 0.17$ \\
\hline
\end{tabular}

Figure I The effect of lapatinib on viability and colony formation in human NPC cells.

Notes: (A) CNE-2 and HONE-I cells were treated with lapatinib (0-5 $\mu$ M) or DMSO control for 48 hours. Phospho-EGFR, EGFR, phospho-HER2, and HER2 were examined by western blotting. GAPDH was used as a loading control. (B) CNE-2 and HONE-I cells were treated with lapatinib (0-10 $\mu$ M) or DMSO control for 24-72 hours. Cell viability was assessed by CCK-8 assay. (C) CNE-2 and HONE-I cells were treated with lapatinib (0-10 $\mu$ M) or DMSO control for 7 days. Colony formation was measured. (D) On the basis of the above experiments, $\mathrm{IC}_{50}$ values are presented as mean \pm standard deviation. 
examined in the presence of increasing concentrations of lapatinib $(0-10 \mu \mathrm{M})$ for different times (24-72 h) using a CCK-8 assay. As shown in Figure 1B, after lapatinib exposure, cell viability was reduced in a dose- and time-dependent manner when compared to the control cells treated with DMSO (vehicle). As shown in Figure 1B, after lapatinib exposure, cell viability was reduced in a dose-dependent manner when compared to the control cells treated with DMSO (vehicle). The assay showed that from 24 to $72 \mathrm{~h}$, the $\mathrm{IC}_{50}$ values of lapatinib in CNE-2 and HONE-1 cells were 3-5 $\mu \mathrm{M}$ (Figure 1D). A colony formation assay was then performed over 7 days, and the colony sizes were reduced by lapatinib in a dose-dependent manner. Moreover, no colonies were detected using the higher dose of lapatinib (Figure 1C).

\section{Induction of apoptosis and autophagy by lapatinib in vitro}

Annexin V-APC/7-AAD double staining was performed to detect apoptosis after lapatinib treatment. Apoptotic activity presenting with the population of Annexin V+ cells gradually advanced with the increasing lapatinib concentration for $48 \mathrm{~h}$ (Figure 2A). In order to better understand the antitumor action of lapatinib, LC3 A/B, a hallmark of autophagy, was measured in the two cell lines in response to lapatinib treatment. A concentration-dependent activation of autophagy was confirmed in both the NPC cell lines, as evidenced by an increase in the level of LC3 B (Figure 2B). Apoptotic activity was further verified by western blot.

\section{Lapatinib activates autophagic cell death via an eEF-2 kinase-dependent pathway}

Lapatinib can trigger apoptosis, and this may account for its antitumor effect. Autophagy is believed to support cell survival and may exert potential cytotoxicity by promoting autophagic cell death. To further explore the role of lapatinib in autophagy, the activity of lapatinib was measured when autophagy was blocked by HCQ, a chemical inhibitor of
A

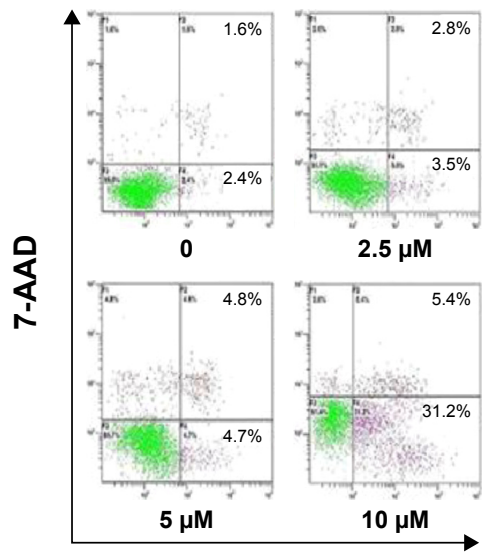

Annexin V-APC

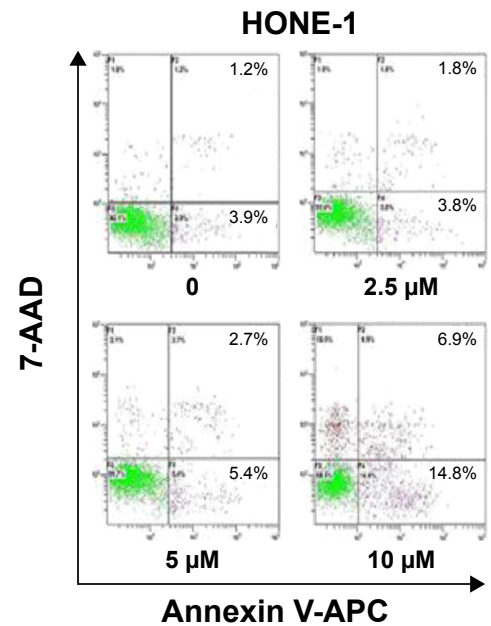

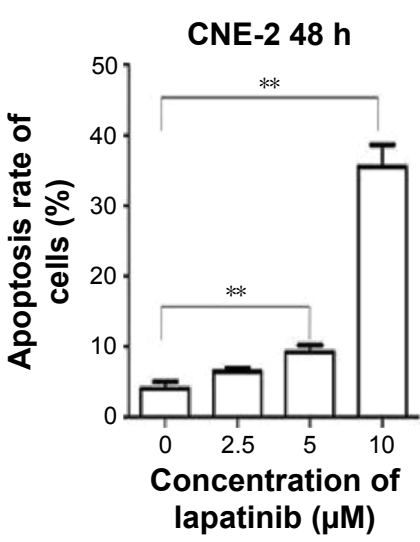

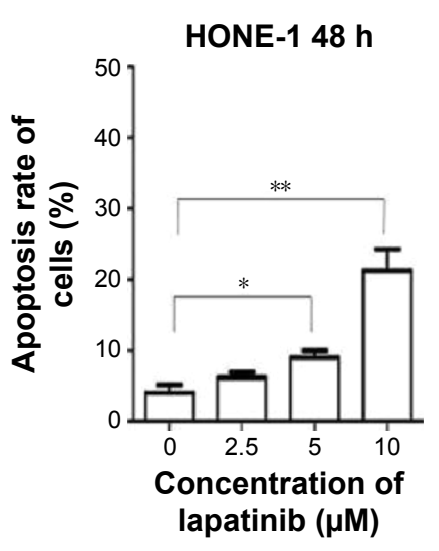

B

CNE-2

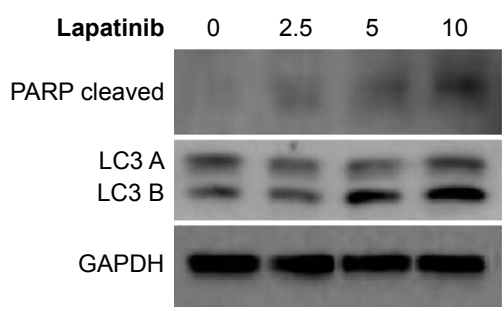

HONE-1

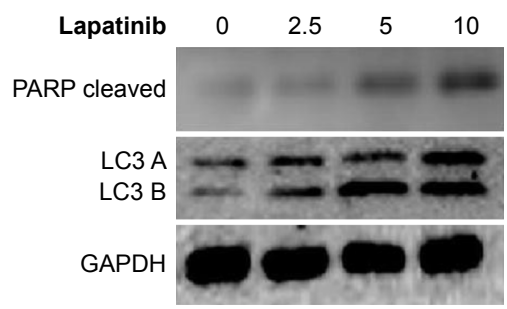

Figure 2 Induction of both apoptosis and autophagy by lapatinib in vitro.

Notes: (A) CNE-2 and HONE-I cells were treated with lapatinib (0-10 $\mu$ M) or DMSO control for 48 hours. Annexin V-APC/7-AAD double staining was performed to detect apoptotic activity, and the results are displayed as histograms. Each bar represents the mean \pm standard deviation. $* P<0.05$; $* * P<0.01$. (b) $C N E-2$ and $\mathrm{HONE}-\mathrm{I}$ cells were treated with lapatinib $(0-10 \mu \mathrm{M})$ or DMSO control for 48 hours. (B) Cleaved-PARP and LC3 A/B were examined by western blotting. GAPDH was used as a loading control. 
A

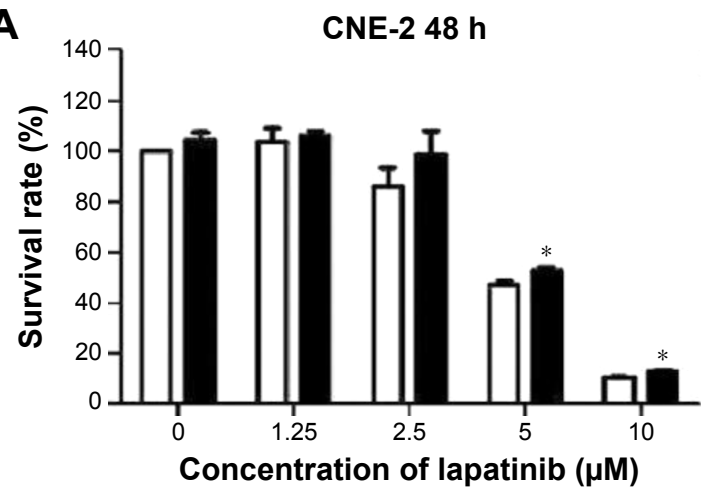

HONE-1 $48 \mathrm{~h}$

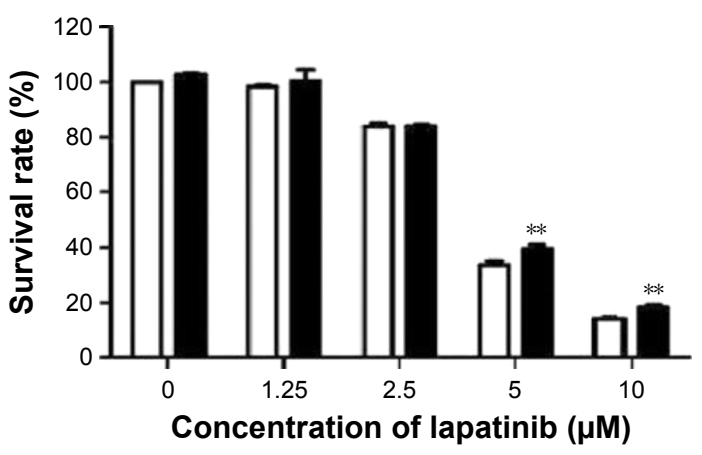

$\square-\mathrm{HCQ} \square+\mathrm{HCQ}$
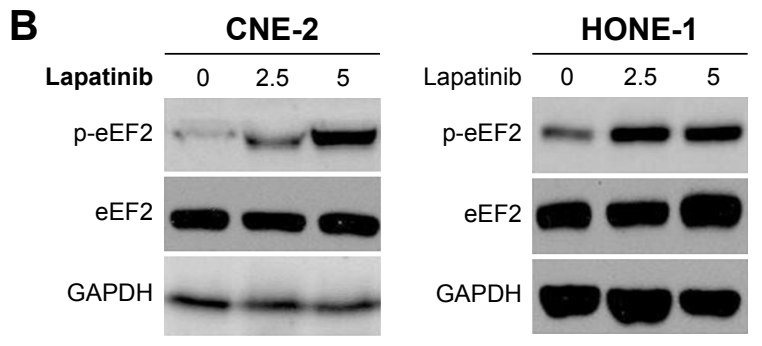

C

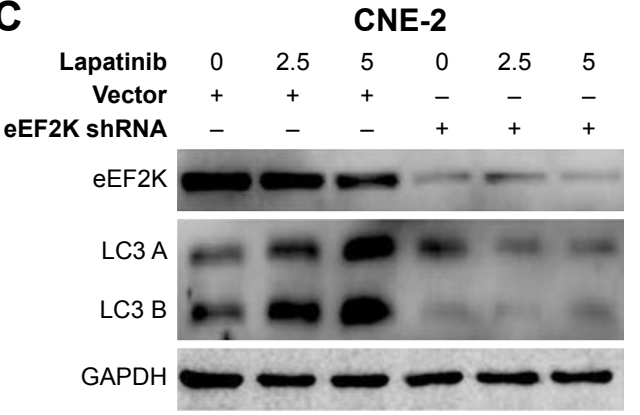

Figure 3 Lapatinib activates autophagic cell death via an eEF-2 kinase-dependent pathway.

Notes: (A) CNE-2 and HONE-I cells were treated with lapatinib (0-10 $\mu \mathrm{M})$ or DMSO control for 48 hours in the presence or absence of $20 \mu \mathrm{M}$ of HCQ. At the end of treatment, cell viability was assessed by CCK-8 assay. Results are shown as the mean \pm standard deviation. $* P<0.05 ; * * P<0.01$. (B) $C N E-2$ and $\mathrm{HONE}-\mathrm{I}$ cells were treated with lapatinib $(0-5 \mu \mathrm{M})$ or DMSO control for 48 hours. Phospho-eEF-2 and eEF-2 were examined by western blotting. GAPDH was used as a loading control. (C) CNE-2 cells were transfected with a non-targeting RNA control (Vector) or a shRNA targeting eEF-2 kinase (eEF2K shRNA), followed by treatment with lapatinib for 48 hours. eEF-2 kinase and LC3 A/B were measured by western blot. GAPDH was used as a loading control.

autophagy. The antitumor effect of lapatinib was markedly decreased by $20 \mu \mathrm{M} \mathrm{HCQ}$ (Figure 3A), suggesting that lapatinib induced autophagic cell death in NPC cells.

Lapatinib activated eEF-2 kinase in a concentrationdependent manner, as indicated by the increased phosphorylation of eEF-2. Autophagy was also induced in NPC cells (Figures 2B and 3B). A gene silencing experiment was then performed to determine the role of eEF-2 kinase in lapatinibinduced autophagy. LC3 B levels decreased when eEF-2 kinase was silenced (Figure 3C), suggesting that lapatinibinduced autophagy requires eEF-2 kinase.

\section{Augmentation of lapatinib-induced autophagy by everolimus enhances the cytocidal effect of lapatinib in NPC cells}

Since lapatinib induced autophagic cell death in NPC cells, whether an autophagy promoter reinforced the antitumor action of lapatinib was evaluated. The mTOR inhibitor everolimus is a major positive regulator of autophagy and acts as a central regulator of the mTOR/S6 kinase/eEF-2 kinase pathway, which plays a regulatory role in the autophagic process in tumor cells. It was observed that the cytotoxicity of lapatinib was significantly increased in the cells treated with $5 \mu \mathrm{M}$ everolimus (Figure 4A and $\mathrm{C}$ ) and that the cytotoxicity of lapatinib was significantly increased in cells treated with 1.25 and $2.5 \mu \mathrm{M}$ everolimus (Figure $4 \mathrm{~A}$ and $\mathrm{C}$ ). In addition, the $\mathrm{IC}_{50}$ of lapatinib was significantly decreased in NPC cells (Figure 4).

A series of western blots were performed to validate the above pathway that mediates eEF-2 kinase during autophagy. As shown in Figure 4C, suppressing mTOR activity led to an augmentation in the amount of LC3 B in the cells compared to lapatinib alone. Lapatinib combined with mTOR inhibitor treatment also resulted in increased activity of eEF-2 kinase (increased phospho-eEF-2) and decreased activity of S6 kinase (decreased phospho-S6 kinase). These results indicate that the mTOR/S6 kinase/eEF-2 kinase pathway is involved in the activation of autophagy by lapatinib and everolimus.

\section{Discussion}

Given the favorable clinical efficacy in advanced HER-2positive breast cancers with lapatinib, a series of preclinical and clinical studies on the efficacy of lapatinib on other HER-2-positive human cancers have been performed. ${ }^{17,18}$ 
A

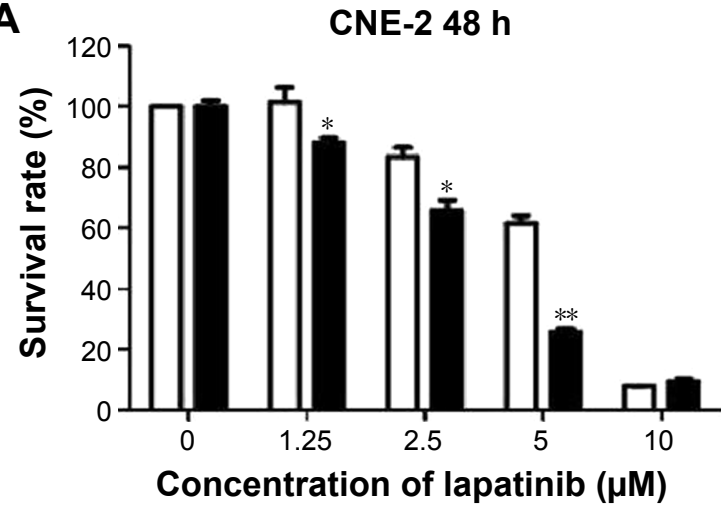

HONE-1 $48 \mathrm{~h}$

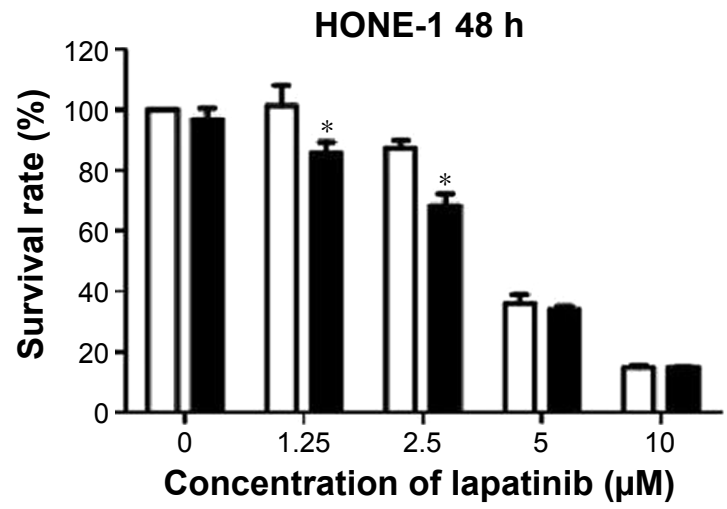

$\square$-Everolimus $\square+$ Everolimus

\begin{tabular}{lll} 
B & & \\
& & \\
\hline $48 \mathrm{~h}$ & IC $_{50}(\mu \mathrm{M})$ & \\
\cline { 2 - 3 } & $\mathrm{CNE}-2$ & HONE-1 \\
\hline -Everolimus & $5.45 \pm 0.17$ & $4.36 \pm 0.09$ \\
+Everolimus & $3.24 \pm 0.17$ & $3.79 \pm 0.22$ \\
\hline
\end{tabular}

C

Lapatinib $\begin{array}{lllllll}0 & 2.5 & 5 & 0 & 2.5 & 5\end{array}$

Everolimus -+++

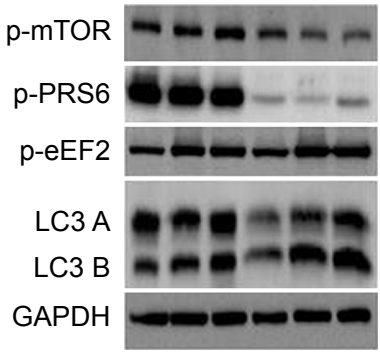

HONE-1

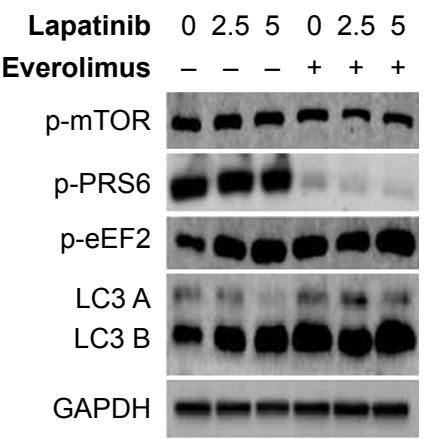

Figure 4 Augmentation of lapatinib-induced autophagy by everolimus enhances the cytocidal effect of lapatinib in NPC cells. Notes: $(\mathbf{A})$ CNE-2 and HONE-I cells were treated with lapatinib $(0-10 \mu \mathrm{M})$ or DMSO control for 48 hours in the presence or absence of $5 \mu$ M of Everolimus. Cell viability was then assessed by CCK-8 assay. Results shown were mean \pm standard deviation. $* P<0.05 ; * * P<0.01$. (B) IC 50 values are presented as the mean \pm standard deviation. (C) CNE-2 and HONE-I cells were treated with lapatinib (0-5 $\mu \mathrm{M})$ or DMSO control for 48 hours in the presence or absence of $5 \mu \mathrm{M}$ of Everolimus, followed by western blotting to examine phospho-mTOR, phospho-PRS6, phospho-eEF2, and LC3 A/B. GAPDH was used as a loading control.

HER-2 and EGFR are coexpressed in many NPC patients in endemic regions of Southeast Asia. ${ }^{19}$ Because of these factors, the therapeutic potential of lapatinib in NPC cells was explored. Lapatinib could significantly inhibit the viability of NPC cells; these results are comparable with other studies in NPC, as well as previous reports in other cancers. ${ }^{1,10}$

The results of the present study showed that lapatinib induced autophagy during the process of apoptosis. Next the role of autophagy in the apoptosis induced by lapatinib was checked. This study suggests that autophagy induction could sensitize NPC cells to lapatinib. Autophagic cell death is also induced in other cancer cell lines, including colon, bladder, and breast cancer cells. ${ }^{20-22}$ Likewise, similar discoveries were reported in hepatocellular carcinoma and chronic myelogenous leukemia K562 cells. ${ }^{12,13}$

Furthermore, it was revealed that eEF-2 kinase (a unique calcium/calmodulin-dependent kinase III) played an important role in regulating lapatinib-induced autophagy. Previous studies have reported that eEF-2 kinase enhances autophagy in response to environmental or metabolic stress. ${ }^{23}$ The observation of this study further confirmed the significance of this kinase in regulating autophagy. However, the molecular mechanisms by which eEF-2 kinase affects autophagy remain poorly understood.

Lapatinib is being tested as a potential cancer therapeutic agent in multiple studies and has been approved for the treatment of breast cancer. Nevertheless, this drug seems to have limited effects as a single agent in NPC. Detecting strategies for increasing lapatinib's induction of autophagy could make this drug more effective in treating cancer. The findings of this study verified that lapatinib-induced autophagy leads to cytotoxicity in tumor cells and that augmenting autophagy could enhance the cytocidal activity of lapatinib. The mTOR lies upstream of eEF2 kinase and can regulate the autophagic response. It is a novel target for the treatment of cancer. Accordingly, we were curious about whether the mTOR inhibitor everolimus could sensitize cells to lapatinib, and the findings revealed that augmentation of lapatinib-induced autophagy by everolimus enhances the cytocidal effect of lapatinib in NPC cells. These results indicate 
that the mTOR/S6 kinase/eEF-2 kinase pathway is involved in the activation of autophagy by lapatinib and everolimus.

The limitations of this study included the use of only two cell lines and the lack of in vivo studies. Also, the synergistic reaction between everolimus and lapatinib was insufficient. Previous research has shown that switching from autophagy to apoptosis can increase AKT inhibitor effectiveness against glioma and that eEF-2 kinase acts as an essential regulator modulating the switch. ${ }^{16}$ In NPC, the associations between autophagy and apoptosis in response to lapatinib remain largely unknown. Thus, further evaluation of the regulation of eEF2 kinase is currently under way.

In summary, augmenting autophagy via eEF-2 kinase activation can modulate NPC cell sensitivity to lapatinib and may be a potential model for combination therapy.

\section{Acknowledgment}

The work was supported by Science and Technology Planning Project of Zhuhai (2012036).

\section{Disclosure}

The authors report no conflicts of interest in this work.

\section{References}

1. Lui VW, Lau CP, Ho K, et al. Anti-invasion, anti-proliferation and anoikis-sensitization activities of lapatinib in nasopharyngeal carcinoma cells. Invest New Drugs. 2011;29:1241-1252.

2. Zhao YY, Tian Y, Zhang J, et al. Effects of an oral allosteric AKT inhibitor (MK-2206) on human nasopharyngeal cancer in vitro and in vivo. Drug Des Devel Ther. 2014;8:1827-1837.

3. Teo PM, Kwan WH, Lee WY, Leung SF, Johnson PJ. Prognosticators determining survival subsequent to distant metastasis from nasopharyngeal carcinoma. Cancer. 1996;77:2423-2431.

4. Lee AW, Ng WT, Chan YH, Sze H, Chan C, Lam CH. The battle against nasopharyngeal cancer. Radiother Oncol. 2012;104:272-278.

5. Ma BB, Lui VW, Poon FF, et al. Preclinical activity of gefitinib in nonkeratinizing nasopharyngeal carcinoma cell lines and biomarkers of response. Invest New Drugs. 2010;28:326-333.

6. Holbro T, Hynes NE. ErbB receptors: directing key signaling networks throughout life. Annu Rev Pharmacol Toxicol. 2004;44:195-217.

7. Kopper L. Lapatinib: a sword with two edges. Pathol Oncol Res. 2008; $14: 1-8$.

8. Ramanathan RK, Belani CP, Singh DA, et al. A phase II study of lapatinib in patients with advanced biliary tree and hepatocellular cancer. Cancer Chemother Pharmacol. 2009;64:777-783.
9. Bekaii-Saab T, Markowitz J, Prescott N, et al. A multi-institutional phase II study of the efficacy and tolerability of lapatinib in patients with advanced hepatocellular carcinomas. Clin Cancer Res. 2009;15:5895-5901.

10. Rusnak DW, Alligood KJ, Mullin RJ, et al. Assessment of epidermal growth factor receptor (EGFR, ErbB1) andHER2 (ErbB2) protein expression levels and response to lapatinib (Tykerb, GW572016) in an expanded panel of human normal and tumour cell lines. Cell Prolif. 2007;40: 580-594.

11. Zhang L, Zhang W, Wang YF, et al. Dual induction of apoptotic and autophagic cell death by targeting survivin in head neck squamous cell carcinoma. Cell Death Dis. 2015;6:e1771.

12. Chen YJ, Chi CW, Su WC, Huang HL. Lapatinib induces autophagic cell death and inhibits growth of human hepatocellular carcinoma. Oncotarget. 2014;5:4845-4854.

13. Huang HL, Chen YC, Huang YC, et al. Lapatinib induces autophagy, apoptosis and megakaryocytic differentiation in chronic myelogenous leukemia K562 cells. PLoS One. 2011;6:e29014.

14. Ryazanov AG, Shestakova EA, Natapov PG. Phosphorylation of elongation factor 2 by EF-2 kinase affects rate of translation. Nature. 1988; 334:170-173.

15. Arora S, Yang JM, Kinzy TG, et al. Identification and characterization of an inhibitor of eukaryotic elongation factor 2 kinase against human cancer cell lines. Cancer Res. 2003;63:6894-6899.

16. Cheng Y, Ren X, Zhang Y, et al. eEF-2 kinase dictates cross-talk between autophagy and apoptosis induced by Akt inhibition, thereby modulating cytotoxicity of novel Akt inhibitor MK-2206. Cancer Res. 2011;71: 2654-2663.

17. Kaufman B, Trudeau M, Awada A, et al. Lapatinib monotherapy in patients with HER2-overexpressing relapsed or refractory inflammatory breast cancer: final results and survival of the expanded HER $2+$ cohort in EGF103009, a phase II study. Lancet Oncol. 2009;10:581-588.

18. Spector NL, Xia W, Burris H 3rd, et al. Study of the biologic effects of lapatinib, a reversible inhibitor of ErbB1 andErbB2 tyrosine kinases, on tumor growth and survival pathways in patients with advanced malignancies. J Clin Oncol. 2005;23:2502-2512.

19. Ma BB, Poon TC, To KF, et al. Prognostic significance of tumor angiogenesis, $\mathrm{Ki} 67$, p53 oncoprotein, epidermal growth factor receptor and HER2 receptor protein expression in undifferentiated nasopharyngeal carcinoma-a prospective study. Head Neck. 2003;25:864-872.

20. Martin AP, Mitchell C, Rahmani M, Nephew KP, Grant S, Dent P. Inhibition of MCL-1 enhances lapatinib toxicity and overcomes lapatinib resistance via BAK-dependent autophagy. Cancer Biol Ther. 2009;8: 2084-2096.

21. Becker MN, Wu KJ, Marlow LA, et al. The combination of an mTORc1/ TORc2 inhibitor with lapatinib is synergistic in bladder cancer in vitro. Urol Oncol. 2014;32:317-326.

22. Cruickshanks N, Tang Y, Booth L, Hamed H, Grant S, Dent P. Lapatinib and obatoclax kill breast cancer cells through reactive oxygen speciesdependent endoplasmic reticulum stress. Mol Pharmacol. 2012;82: 1217-1229.

23. Cheng Y, Li H, Ren X, Niu T, Hait WN, Yang J. Cytoprotective effect of the elongation factor-2 kinase-mediated autophagy in breast cancer cells subjected to growth factor inhibition. PLoS One. 2010;5:e9715.
OncoTargets and Therapy

\section{Publish your work in this journal}

OncoTargets and Therapy is an international, peer-reviewed, open access journal focusing on the pathological basis of all cancers, potential targets for therapy and treatment protocols employed to improve the management of cancer patients. The journal also focuses on the impact of management programs and new therapeutic agents and protocols on

\section{Dovepress}

patient perspectives such as quality of life, adherence and satisfaction. The manuscript management system is completely online and includes a very quick and fair peer-review system, which is all easy to use. Visit http://www.dovepress.com/testimonials.php to read real quotes from published authors. 